\title{
Les métaphores de la séduction dans les journaux des marins français à Tahiti en avril 1768
}

Les métaphores de la séduction dans les journaux des marins français à Tahiti en avril 1768

\section{Anaïs de Haas}

\section{(2) OpenEdition}

\section{Journals}

Édition électronique

URL : http://journals.openedition.org/jso/7200

DOI : $10.4000 /$ jso. 7200

ISSN : $1760-7256$

\section{Éditeur}

Société des océanistes

Édition imprimée

Date de publication : 15 décembre 2014

Pagination : 175-182

ISBN : 978-2-85430-118-2

ISSN : 0300-953x

\section{Référence électronique}

Anaïs de Haas, "Les métaphores de la séduction dans les journaux des marins français à Tahiti en avril 1768 », Journal de la Société des Océanistes [En ligne], 138-139| 2014, mis en ligne le 15 décembre 2017, consulté le 20 avril 2019. URL : http://journals.openedition.org/jso/7200 ; DOI : 10.4000/ jso.7200 


\section{Les métaphores de la séduction dans les journaux des marins français à Tahiti en avril 1768}

par

Anaïs de HAAS*

\section{RÉSUMÉ}

Lors du voyage de Bougainville autour du monde, plusieurs marins ont tenu des journaux de bord, qui sont particulièrement prolixes pour décrire l'escale à Tahiti. Pour raconter l'étrange accueil que leur ont fait les Tahitiens, les marins utilisent des métaphores relevant alternativement de la Bible, de la mythologie grecque ou des topoï libertins. Cet article propose une analyse de ces différents réseaux métaphoriques, des modalités selon lesquelles ils s'articulent, des phénomènes sémiotiques qui les motivent et de ce qu'ils révèlent sur la pratique gnoséologique bien particulière du "voyage de découverte".

Mots-CLÉs : Tahiti, Bougainville, journaux de bord, équivocité, métaphores

En avril 1768, l'Étoile et la Boudeuse ont jeté leurs ancres pour dix jours dans la baie de Hitiaa, à Tahiti. Plus de trois cents hommes voyageaient sur ces deux navires. Six journaux de bord rédigés au cours de ce voyage ont été retrouvés, transcrits et édités par Étienne Taillemite en 1977 : celui du capitaine Louis-Antoine de Bougainville, du lieutenant Jean-Louis Caro, du chirurgien François Vivez, du botaniste Philibert Commerson et des deux jeunes volontaires, Charles-Félix-Pierre Fesche et Charles-Henri-Nicolas-Othon de Nassau Siegen (Taillemite, 1977). Cette escale à Tahiti est un épisode particulièrement fascinant du voyage, qui continue de susciter interprétations et controverses.

Dès le premier jour de l'arrivée des navires français, l'accueil est plus que cordial. Les Tahitiens

\begin{abstract}
During the circumnavigation of Bougainville, several navigators were writting logbooks, which are particulary descriptive of the stopover they made in the island of Tahiti. To describe the strange way in which they were welcomed by the Tahitians, the navigators were using metaphors linked alternatively with the Bible, the Greek mythology or the libertine topoï. This article aims to analyse these different metaphorical networks, the ways they are organised, the semiotical phenomenons that cause them, and what they reveal about the particular knowledge practice of the "discovery journeys".
\end{abstract}

KeYwords: Tahiti, Bougainville, logbooks, equivocity, metaphors

arrivent sur leurs pirogues jusqu'aux navires, pour offrir aux Français de la nourriture en abondance. Puis, très vite, les Tahitiens et Tahitiennes vont faire comprendre aux marins qu'on attend d'eux qu'ils aient des relations sexuelles avec les femmes... Les métaphores utilisées par les marins dans leurs journaux pour raconter ces rencontres sexuelles méritent une étude attentive. On propose d'analyser ici un récit dû à Fesche, jeune volontaire de vingt-trois ans. Voici l'extrait, dans sa graphie d'origine:

« Nous étions trois ensemble, nous partons à dessein de nous promener escortés d'une troupe d'insulaires, nous arrivons dans une case où nous sommes bien reçu par le maitre de la maison, d'abord il nous fait voir ses possessions, nous faisant entendre qu'il

\footnotetext{
* Centre de Linguistique anthropologique et sociolinguistique, Institut Marcel Mauss, EHEss. anais.dehaas@ehess.fr
} 
attendoit ses femmes qui devoient arriver dans peu. [...] Après quelque temps de promenade, nous retournâmes à son logis où nous trouvons sa femme et une jeune fille de 12 à 13 ans. On nous fait asseoir, on apporte des cocos, des bananes, on nous invite à manger, nous les satisfaisons. Nous voyons ensuite chacun d'eux s'emparer d'une branche de feuillage et se ranger en cercle autour de nous, un des assistans s'empara d'une flûtte dont il en tira des sons doux et agréables et on apporta une natte que l'on étendit sur la place et sur laquelle s'assit la jeune fille. Les signes de tous les Indiens nous firent bien comprendre le fait dont il s'agissoit, cependant cet usage étant si contraire à ceux établis pour nous et voulant en être assuré, un de nous s'approche de la victime offerte, lui fait présent d'une fausse perle qu'il lui attache à l'oreille, risque un baiser qui lui fut bien rendu. Une main hardie et conduite par l'amour se glisse sur deux pommes naissantes ennemies l'une de l'autre et dignes comme celles d'Heleine de servir de modèle à des coupes incomparables par la beauté et l'agrément de leur formes. La main glissa bientôt et par un heureux effet de hasard, tomba sur des appas cachés encore par une de leur toille, elle fut bientôt otée par la fille elle-même que nous vîmes alors avec le seul habillement que portoit Ève avant son péché. Elle fit plus, elle s'étendit sur la natte, frappa sur la poitrine de celui qui étoit son agresseur, lui faisant entendre qu'elle se donnoit à lui et écarta ces deux obstacles qui empêchent l'entrée de ce temple où tant d'hommes sacrifient tous les jours. L'appel étoit bien engageant et l'athlète qui la caressoit connoissoit trop bien l'art de l'excrime pour ne pas la prendre sur le temps si la présence de 50 Indiens qui l'environnoient n'eut, par un effet de nos préjugés, mis un frein à ses désirs violents, mais quelque ardeur qui vous anime, il est bien difficile de surmonter tout d'un coup les idées avec lesquelles on a été nourri. Après quelque temps de séjour dans cette case, nos yeux las de regarder et de toucher, nous nous retirâmes, les habitants forts mécontents de nous voir aussi peu âpre à la curée et même nous le témoignant. " (Journal de Fesche, in Taillemite, 1977 : tome I, 81)

Outre l'aspect déroutant des événements relatés, c'est bien le foisonnement des réseaux isotopiques qui surprend et amuse. Cet écheveau métaphorique, que l'on va tenter de démêler, caractérise en fait l'ensemble des pages des journaux concernant l'escale à Tahiti.

\section{« Ève », le « sacrifice " et l' " art de l'escrime " : fixer de vertigineuses incompréhensions}

Notons tout d'abord le recours fréquent à des périphrases : "le fait dont il s'agissoit ", "le seul habillement que portoit Ève avant son péché ", "l'entrée de ce temple où tant d'hommes sacrifient tous les jours "... Ces détours peuvent bien-sûr être expliqués par la bienséance de ce jeune homme courtois du XVIII ${ }^{\mathrm{e}}$ siècle, pour qui les mots précis de la sexualité ne viennent pas spontanément à la plume. Néanmoins ces péri- phrases nous semblent témoigner aussi d'une motivation du discours, d'une licence de l'expressivité. Fesche ne se limite pas à une description efficace et succincte, mais laisse son langage libre de se déployer : ce récit ne se borne pas à rendre compte, il veut vraiment raconter.

Pour raconter le corps de la jeune Tahitienne, dans le passage qu'on a retranscrit, Fesche mobilise deux figures de femmes mythologiques: Ėve et Hélène. Les mythologies biblique et antiques constituent en fait un paradigme récurrent dans toutes les pages des journaux concernant cette escale. Les jeunes et belles Tahitiennes sont très fréquemment évoquées par les noms Ėve, Hélène et Vénus, et ces antonomases sont les termes de départ d'une expansion synecdotique qui englobe le lieu tout entier dans cette isotopie de l'amour. L'île qui avait été de prime abord baptisée par Bougainville "Mont de Bourbon " devient ainsi très rapidement sous les plumes des diaristes "l'isle de Cythère ", " l'isle de l'Amour ", « le jardin d'Eden », "l'isle de Vénus ».

Ces recours aux figures les plus importantes de la cosmogonie occidentale montrent tout d'abord, nous semble-t-il, que les marins ont été fortement impressionnés par les événements auxquels ils étaient confrontés. Pour autant, ni les métaphores édéniques ni les métaphores mycéniennes ne parviennent à contenter leurs aspirations descriptives: dans ce réservoir paradigmatique, les images bibliques et antiques sont invoquées comme un leitmotiv, mais indistinctement les unes des autres... preuve que ni la Bible ni les dieux de la Grèce Antique ne parviennent à épuiser, à fixer la fascination qu'exerce sur eux Tahiti.

Alors, évidemment, ces pages des journaux montrent que les marins ont compris "de travers" la cosmologie Tahitienne, puisqu'ils invoquent leur propre mythologie pour rendre compte des comportements étrangers qui n'y sont reliés d'aucune manière. Mais à ce reproche, on peut répondre d'une part que les discours des marins laissent entrevoir qu'eux-mêmes ne sont pas satisfait de ces descriptions, et d'autre part qu'ils avaient tout de même eu l'intuition que ces événements sexuels relevaient de quelque chose de très fortement symbolique, de quelque chose de mythique. En effet, outre les références mythologiques, on trouve dans notre extrait le mot " temple ", le verbe "sacrifier ", et dans d'autres passages le mot "culte " ("culte à Vénus »).

Notre hypothèse est donc que les réseaux métaphoriques actualisés par les marins ne sont ni des purs fantasmes - ils sont attentifs aux références réelles - , ni un plaquage ethnocentrique négligeant la différence de l'altérité tahitienne les mythologies occidentales sont invoquées par défaut - mais plutôt la manifestation discursive d'un grand désarroi dans leur effort de fixation de ces vertigineuses incompréhensions. 
Ainsi, quand les marins recourent aux concepts d' « amour » et de « mariage " pour signifier ces événements, il faut voir dans ces métaphores exactement ce qu'a décrit Roy Wagner dans The Invention of Culture. Pour surmonter le « choc culturel » - un sentiment d'inadéquation, de perte de contrôle - , ils tentent de fixer dans leur propre langage les choses qu'ils ne peuvent fixer, qu'ils ne peuvent contrôler autrement (Wagner, 1975). On pourrait peut-être même interpréter comme un paroxysme du désir de fixation, dans l'extrait cité de Fesche, cet étrange éloge des seins de la jeune fille :

" deux pommes [...] dignes comme celles d'Heleine de servir de modèle à des coupes incomparables par la beauté et l'agrément de leur formes. »

Fesche, dans son admiration, voudrait transformer en coupes, c'est-à-dire en objet, l'anatomie de la troublante jeune tahitienne... Dans Métaphysiques cannibales, Eduardo Viveiros de Castro a analysé les modalités de connaissance de la modernité occidentale comme un effort de désubjectivation - en contraste avec le chamanisme amérindien où la connaissance passe par la subjectivation, la révélation de l'intentionnalité - : la remarque de Fesche semble une illustration saisissante de cette volonté d'objectivisation (Viveiros de Castro, 2009).

Un autre réseau métaphorique remarquable dans les pages des journaux relatives à cette escale, déjà largement perceptible dans l'extrait cité ici, est celui du combat : l'escrime, le risque, la hardiesse, l'agresseur et la victime sont tour à tour évoqués pour décrire les gestes du marin face à la jeune fille. Ces métaphores guerroyantes relèvent en fait de l'isotopie du libertinage. Sade, Prévost, décrivent eux aussi le séducteur comme un stratège, qui doit enjôler ou piéger sa "victime". Mais dans ce contexte, lors de cette escale des Français, ces formules semblent tout à fait hors de propos : les femmes n'ont absolument pas besoin d'être " piégées ", puisque toute la société tahitienne les incite à s'offrir d'elles-mêmes...

Alors pourquoi les marins utilisent-ils malgré tout la métaphore du séducteur stratège et guerrier? Une hypothèse psychologique apparaît : puisqu'au moment des événements les rôles du topos libertin du XVIII ${ }^{\mathrm{e}}$ siècle sont inversés - la femme tahitienne s'offre, et quand l'homme français n'obtempère pas on lui en fait le reproche -, au moment de l'écriture les marins pourraient avoir eu tendance à charger leur récits d'images de séducteurs-stratèges, pour rééquilibrer leurs ego masculins mis à mal... D'autant plus que nos marins ont avoué à plusieurs reprises leur anxiété à ce sujet. Ainsi Fesche, à la suite de l'extrait cité, tient à assurer à ses lecteurs que : «plusieurs françois, moins susceptibles de délicatesse, le même jour, ont trouvé plus de facilité à lever les préjugés "; et encore, après un autre épisode similaire :

« cette seule occasion suffisoit pour donner une mauvaise idée de la galanterie et de la bouillante ardeur si généralement reconnu dans les françois si le séjour fait dans cette isle que nous avons nommé la Nouvelle Cythère à cause des mœurs de ses habitants ne nous eut procuré l'occasion d'effacer amplement la mauvaise opinion qu'il devoient avoir conçu de nous. "

Le récit du faux combat entre la femme qui veut et l'homme qui cherche à la piéger donne ainsi lieu à des juxtapositions antithétiques tout au long de l'extrait : "victime / offerte », " risque un baiser / qui lui fut bien rendu ", " une main hardie / et conduite par l'amour »... Quand on a pris conscience de ces antinomies, la dernière, la fin de l'extrait, résonne d'autant plus comiquement :

"L'appel étoit bien engageant et l'athlète qui la caressoit connoissoit trop bien l'art de l'excrime pour ne pas la prendre sur le temps si la présence de 50 Indiens qui l'environnoient n'eut, par un effet de nos préjugés, mis un frein à ses désirs violents, mais quelque ardeur qui vous anime, il est bien difficile de surmonter tout d'un coup les idées avec lesquelles on a été nourri. »

Le début de la période est pompeusement cadencée, la protase vante l'adresse du combattant, crée un suspens avec l'acmè sur le mot «temps »...puis l'apodose rappelle le contexte la présence de tout le village autour du couple - , révèle comme toute cette mise en scène discursive est factice, et tente d'éluder cette contradiction dans un touchant sursaut de justification - « mais »...

\section{Embarras sexuel, désarroi social, instabilité discursive}

L'emphase des discours des marins relatant les rencontres sexuelles à Tahiti a donné naissance à un " mythe " occidental sur la sexualité à Tahiti, qui ne correspondait pas exactement à la réalité, certes. Mais plutôt que d'expliquer les causes de cette exagération par l'androcentrisme et le racisme des marins ${ }^{1}$, il nous semble pertinent d'interpréter cette emphase comme résultant de leur

1. Cette perspective a été argumentée par Serge Tcherkézoff (2005). Ce livre fait appréhender toute la complexité des événements et des interprétations qui se sont joués à Tahiti lors de ces dix jours, et démontre les biais occidentaux et masculins dans la manière par laquelle les femmes tahitiennes ont été perçues et décrites. Nous pensons néanmoins que, pour analyser les biais « sexistes " des discours des marins par rapport à la réalité des événements, il faut aussi être attentifs à la possibilité que les événement aient tourmenté les marins eux-mêmes - car, tout de même, ce que l'extrait du journal Fesche cité ici finit par raconter, c'est une impuissance sexuelle. 
besoin de se justifier par rapport aux normes de la virilité de la société occidentale - éventuellement aussi par rapport aux attentes de la société tahitienne, comme on va le voir -, et d'atténuer ainsi leur désarroi après des événements pour le moins troublants.

Ces embarras sexuels semblent en effet être interprétés par les Tahitiens comme une inconvenance politique, voire comme une incongruité cosmologique. Marshall Sahlins, pour expliquer l'ire des Hawaïens provoquée par le retour sur leur île du navire du capitaine Cook, après un premier départ avorté du fait d'une avarie du mât, écrit :

«le problème n'était ni empirique ni pratique : il était cosmologique - et, à cet égard, l'état du mât du Resolution était proprement inintelligible. "(Sahlins, 1989)

Un même type d'incommensurabilité a probablement caractérisé les rencontres sexuelles inabouties, à Tahiti en avril 1768 .

Dès le premier jour, Fesche évoquait le mécontentement des Tahitiens face aux attitudes trop scrupuleuses des Français :

"Cette nouvelle Vénus, après avoir longtemps attendu, voyant que ni les invitations de ses concitoyens et principalement de ses vieillards, ni l'envie qu'elle témoignait elle-même d'offrir avec un de nous quel qu'il fut un sacrifice à Vénus, ne pouvoient nous engager à transgresser les règles de la décences et des préjugés établis pour nous, sentiment qu'elle interprétait peut-être à notre désavantage, nous quitta d'un air piqué et se sauva dans sa pirogue. » (Journal de Fesche, in Taillemite, $1977:$ t. 1, 80)

Mais là où le marin fait l'inférence d'un jugement sur sa virilité, et s'en inquiète comme tel, le mécontentement de la Tahitienne prend lieu sur une autre dimension.

On aura pu remarquer encore une fois, dans ce dernier extrait, une juxtaposition antithétique: " un air piqué " / " se sauva ». Par l'adjectif "piqué ", par le contexte discursif dans lequel il s'insère, Fesche nous dit que la femme est énervée parce qu'elle n'a pas obtenu la relation sexuelle qu'elle escomptait. Et pourtant, avec le syntagme verbal suivant " et se sauva dans sa pirogue ", il essaie de nous en donner une toute autre image : l'image d'une ingénue qui s'enfuirait pour échapper à son séducteur, au piège qu'il lui aurait tendu... Cette amusante discordance nous semble montrer encore une fois que le désarroi sexuel, social, ontologique que subissent les marins est le moteur principal de la versatilité discursive dans les journaux, du foisonnement et de la variété des métaphores.

"Amour ", " mariage ", " culte ", " sacrifice ", " hospitalité "... : tous ces concepts occidentaux ont été tour à tour invoqués dans les journaux pour tenter d'interpréter l'incommensurable étrangeté des requêtes sexuelles de la société tahitienne aux marins français. Encore une fois, l'hétérogénéité de l'ensemble montre que les emplois de ces termes n'expriment pas un jugement ferme, assuré et satisfait ${ }^{2}$.

Lorsque Bougainville, dans le Voyage - version retravaillée de son journal de bord - raconte la célèbre mésaventure de son cuisinier à l'arrivée à Tahiti, on retrouve la même alternance entre différents registres. Bougainville présente d'abord la séduction de la jeune fille comme langoureuse - elle laisse tomber "négligemment" le pagne qui la couvrait...- et le cuisinier comme un séducteur audacieux - il " avait trouvé le moyen de s'échapper" "malgré les défenses " - avant que le situation ne se renverse, et que le pauvre cuisinier ne remonte sur le bateau totalement apeuré : la foule tahitienne l'avait déshabillé et avait "fait approcher la fille en le pressant de contenter les désirs qui l'avaient amené à terre avec elle » (Bougainville, 1982 : 226).

La manière de se référer à la phraséologie particulière de leur époque pour mettre en mots ce qu'ils vivent, ainsi que le travail de ré-écriture que l'on remarque en étudiant les différentes versions des journaux, tout cela montre selon nous que les marins étaient en proie à un sentiment d'insécurité pas seulement physique mais, surtout, sémiotique.

\section{Bricolage sémiotique}

Les Tahitiens parlaient une langue inconnue, vivaient dans un autre monde que celui des Français : évidemment, ces derniers, en dix jours, n'ont pas pu comprendre les motivations

2. C'est pourquoi nous trouvons problématique la manière dont Tcherkézoff analyse l'occurrence du terme « hospitalité ». Bougainville, pour parler de ce qui se passe quand de jeunes Tahitiennes sont présentées aux marins, écrit : "on a célébré l'hospitalité » et la métaphore se retrouve aussi dans d'autre journaux. À partir de cela, Tcherkézoff analyse : "Les voyageurs [...] n'ont vu dans les jeunes femmes polynésiennes que des courtisanes [...]. ", " [Bougainville] assimile cette offrande de jeune fille au contexte de l'homme européen séduisant une femme dans une auberge et recherchant alors une intimité pour assouvir son désir. Incapable de relativiser le contexte et de voir qu'il s'agit d'un rituel, peut-être même d'un rite sacrificiel, et non d'une passade dans une auberge, il ne lui reste qu’à relativiser la coutume de l'hospitalité. » $(2005: 202)$. Bien sûr, Bougainville - et les autres -, pour signifier ce qu'ils voient et ce qu'ils vivent, essaient des analogies avec leurs propre concepts : ici l'hospitalité. Mais les marins auteurs des journaux ne nous paraissent pas se sentir comme s'ils étaient chez eux, "séduisant une femme dans une auberge ». Il nous semble rapide et excessif de voir dans la métaphore que fait Bougainville une " assimilation ". Considérer uniquement cette analogie, et la critiquer comme un jugement définitif, qui alimenterait ainsi le réquisitoire contre le "sexisme » des marins, c’est risquer de manquer toute une étape de leur expérience. 
de leurs comportements. Pourtant, à partir du moment où ces six diaristes prenaient la plume et se penchaient sur leurs cahiers, ils prenaient dans le même temps la décision de donner un sens aux événements auxquels ils avaient assisté ou participé. En 1768, les questionnements réflexifs des sciences humaines ne constituaient pas un arrière plan méthodologique habituel comme il peut l'être aujourd'hui. En outre, les marins n'écrivaient pas des journaux intimes: les mots qu'ils calligraphiaient étaient destinés à être lus par leur familles, amis - et pour le roi en ce qui concerne le journal de Bougainville. Dans les journaux de bord, il ne s'agissait donc pas pour les marins de discourir sur leurs capacités personnelles à expliquer ou non les événements et la culture tahitienne. Il fallait avant tout raconter, signifier, cette île et ses habitants que personne en Europe ne connaissait encore.

Quand Tcherkézoff analyse le "mythe occidental de la sexualité polynésienne", le terme « mythe» est connoté péjorativement: les marins auraient "inventé ", auraient " tout déformé » (2005), ils auraient initié la divulgation d'une fausse idéologie. Nous voudrions quant à nous, à partir de ces analyses linguistiques, considérer l'autre aspect de la polysémie du terme "mythe». Car, en somme, cette interprétation occidentale de Tahiti constitue un récit invoqué pour comprendre des phénomènes qui échappent aux efforts directs de description : l'anthropologie structurale pourrait ne pas désavouer cette définition de " mythe".

Considérer les écrits des marins non comme un mythe-mensonge, mais, moins sévèrement, comme un mythe-explication du monde, permet de penser autrement les phénomènes d'interprétation en jeu lors de cette interaction. D'abord, les marins diaristes se réferent à leur propre mythologie antique et biblique, et aux topoï du libertinage, pour pouvoir percevoir, signifier, pour pouvoir parler de cette situation étrange dans laquelle ils sont. Ensuite, ce faisant, ils participent effectivement, malgré eux, à la création de quelque chose comme un mythe : l'allégorie de Tahiti comme l'île de l'Amour.

Cet angle d'analyse permet en outre de concevoir une symétrie entre, d'une part, le "mythe" Tahitien concernant la toute puissance de ce qui arrive de la mer, et, d'autre part, le "mythe " à travers lequel les Français ont appréhendé les Tahitiens. Selon Marshall Sahlins, toute culture contient - est capable d'expliquer - le monde entier. Les cultures « sont toujours universelles dans leur portée, et, par là même, capables d'intégrer des objets et des personnes étrangères dans des relations logiquement cohérentes "; " toute société connue de l'histoire est une société globale, chaque culture un ordre cosmologique [...], englobant l'univers entier dans son propre schème culturel " (2007 : 287). Dans cette perspective, les récits des marins témoigneraient ainsi moins d'une "invention " inique et condamnable, que de la manifestation d'un invariant anthropologique : en nous racontant d'un côté la manière dont les Tahitiens se comportent avec eux, et de l'autre côté la manière dont eux-mêmes perçoivent les indigènes, les journaux des marins nous donnent à voir deux manières d'interpréter l'Étranger mises en face à face, deux bricolages sémiotiques, émanant d'un phénomène universel.

\section{Les métaphores des marins comme parole " sauvage"}

Lévi-Strauss, dans La Pensée sauvage propose une distinction entre la "pensée domestiquée", se définissant par un projet, une logique a priori, et la "pensée sauvage " où une logique se développe a posteriori. La première produit des événements par l'expérimentation, par une volonté consciente et dans le but de faire progresser la connaissance ou de rentabiliser des phénomènes; la seconde consiste à expliquer les événements du présent par d'autres événements passés, faisant ainsi advenir de l'intelligible par l'agencement implicite d'éléments concrets. L'expérimentation scientifique, telle que nous la connaissons en Occident, est ainsi le parangon de la pensée domestiquée, alors que les mythes sont des manifestations archétypales de la pensée sauvage. Ces deux types de pensées n’ont pourtant pas une valeur équivalente : la pensée sauvage - ou pensée mythique - semble être forcément antérieure, puisqu'elle serait «la condition de toute pensée ", la modalité première de connaissance (Lévi-Strauss, 1962).

Translatée sur le plan des discours, cette distinction pourrait nous faire mieux comprendre deux tendances expressives, deux modes de description qui s'opposent singulièrement dans les pages des journaux concernant Tahiti.

Rappelons-nous qu'une des ambitions de cette expédition dirigée par Bougainville était explicitement la "découverte»: augmenter - voire même achever! - la connaissance de la géographie, de la flore, de la faune, et des sociétés humaines. La méthode naturaliste et taxinomique est employée pour ce projet : collecter des données sur les phénomènes ou les réalités rencontrées, en ramenant tout le système du visible à un système de variables, dont toutes les valeurs peuvent être assignées, soit par un chiffre, soit par une description méthodique et finie, dont les critères ont été établis a priori. Projet, logique a priori, engrangement de connaissances positives: les passages des journaux qui exploitent cette méthode taxinomique relèvent clairement de la "pensée domestiquée ". 
À l'inverse, les descriptions plus libres, là où abondent les métaphores qui bricolent du sens à partir d'éléments préexistants dans le réservoir mythologique occidental, manifestent l'activité d'une pensée "sauvage». Les taxinomies sont conscientes, voulues, prévues. Les métaphores paraissent fortuites, contingentes, et pourtant inévitables à partir du moment où les marins ont fait le choix de parler de ce qui les trouble.

Ces manifestations linguistiques de deux types de pensée différents procèdent pourtant d'une même exigence intellectuelle de signification. Selon Lévi-Strauss, l'attribution d'une signification précède la connaissance de cette signification :

"l'homme dispose dès son origine d'une intégralité de signifiants, dont il est fort embarrassé pour faire l'allocation à un signifié, donné comme tel sans pour autant être connu. » (Lévi-Strauss in Marcel Mauss, 1950)

Le projet taxinomique permet de résorber positivement cet excès de signifiant, qui était, en quelque sorte, prévu : les taxinomies, les noms, les cases, étaient déjà tout prêts, et ne demandaient qu'à se remplir des nouveaux signifiés qu'on allait rencontrer. En revanche, l'usage de métaphores marque une résorption moins heureuse : elle prouve que le rapport d'inadéquation entre l'excès de signifiant à disposition et le signifié empirique n’a pas pu être comblé.

Il nous semble que nous pouvons voir là deux usages différents du langage : la parole « domestiquée » des taxinomies montre un projet de langage, quand la parole « sauvage » des métaphores montre l'usage du langage comme d'un instrument, d'un outil imparfait, exploité par dénuement, par absence d'autre possibilité.

Partis avec la volonté de déployer une signification hégémonique, avec l'espoir positiviste d'un langage infaillible, les marins nous montrent ainsi, par leurs écrits, qu'ils ne peuvent pas ne pas passer par le bricolage de la pensée "sauvage ».

\section{Singularités de la pratique gnoséologique occidentale}

Pour autant, alors que le «bricolage sémiotique " semble être un processus commun, les modalités selon lesquelles d'une part les Tahitiens, d'autre part les marins français, l'ont déployé lors de cette rencontre different. Les Tahitiens avaient, semble-t-il, " prévu ", interprété à l'avance, l'arrivée des Européens. Quand ces derniers sont arrivés, d'après ce que l'on peut lire dans leurs journaux, les indigènes semblaient assez assurés de ce qu'ils devaient faire face à cet événement, bien qu'ils ne l'aient pas provoqué activement.
En contraste, les circumnavigations depuis la France et l'Angleterre, menées successivement par John Byron (1764-1766), Samuel Wallis (1766-1768), Louis-Antoine de Bougainville (1767-1769) et James Cook (1768-1771, 17721775 et 1776-1780) témoignent d'une volonté de signification prospective dans l'espace. Soutenus et financés par George III et Louis XV, ces voyages n'étaient pas seulement des initiatives individuelles, mais des ambitions qui engageaient les nations. La modernité occidentale a ainsi projeté ses voyages et sa "libido interpretandi » jusqu'à l'autre bout du monde. Voilà la modalité selon laquelle les royaumes français et anglais de la double décennie 1760-1770 ont englobé l'univers entier dans leur propre schème culturel : en le caractérisant comme de nouvelles choses à connaître, et qu'il faut aller voir pour connaitre.

Une fois les bateaux arrivés, au cours de l'interaction, le choc culturel n'a pas non plus été négocié de la même manière selon chaque partie. Comment les Tahitiens ont-ils géré le fait que les marins étaient autres, différents ? En leur apportant de la nourriture, en voulant avoir des relations sexuelles avec eux. Comment les marins ont-ils, eux, géré l'altérité tahitienne? En voulant la raconter par écrit. L'écriture a pour cela une importance majeure, puisqu'elle synthétise d'une part la fixation par le langage, notamment au travers du phénomène de métaphorisation décrit par Roy Wagner, et d'autre part une matérialisation de cette parole, qui, servant une volonté d'objectivation, permet la conservation, l'enregistrement. Du fait de l'écriture, les modalités abstraite et concrète de ce désir de fixation se rejoignent... jusqu'à aujourd'hui, où la tradition archivistique se poursuit.

Ainsi, le fait que l'on puisse aujourd'hui consulter les manuscrits originaux de journaux des marins n'est pas seulement ce qui permet de continuer leur analyse, mais est tout à fait en continuité avec le fait que les marins aient écrit ces journaux. À l'inverse, le fait qu'il n'existe pas de traces écrites relatant ces événements du point de vue des Tahitiens n'est pas une absence regrettable - même si, bien-sûr, de notre point de vue exégétique on aurait rêvé pouvoir faire la comparaison avec ce qu'auraient pu être les écrits tahitiens. Mais si la société tahitienne avait été une société à écriture, son ontologie n'aurait pas été la même, les événements n'auraient pas été les mêmes, et peut-être n'auraient-ils pas permis de faire prendre conscience de l'usage particulier que l'Occident a fait du langage lors de ces circonstances.

La vocation ultime du désir de connaissance dont les marins étaient investis, c'était donc la trace écrite. Les marins pensaient arriver à mieux connaître les Tahitiens non pas en les mangeant, non pas en les tuant, non pas en ayant des relations 
sexuelles avec eux, mais bien en traçant des circonvolutions d'encre sur des feuilles de papier. Considérer cette escale d'avril 1768 en projetant les jugements inspirés par la critique de la colonisation, c'est non seulement risquer d'infléchir l'analyse des discours des marins $s^{3}$, mais aussi, paradoxalement, risquer de dévitaliser la pensée de l'indigène dont on veut prendre la défense ${ }^{4}$. Certes, la modalité gnoséologique occidentale - en tant que structure de pensée - et la colonisation - en tant qu'événement - sont sûrement liées. Mais plutôt que de les assimiler, la critique évidemment nécessaire de la seconde pourrait être affinée et enrichie par l'étude de la première : par une heuristique des malentendus lors des rencontres provoquées par les voyages de "découverte».

\section{BIBLIOGRAPHIE}

Bougainville Louis-Antoine, 1982. Voyage autour du monde, Paris, Gallimard.

LÉvi-Strauss Claude, 1950 (2010). Introduction à l'œuvre de Marcel Mauss, in $\mathrm{M}$.
Mauss, Sociologie et anthropologie, Paris, PUF, pp. IX-LII.

—, 1962. La pensée sauvage, Paris, Plon.

SaHLins Marshall, 1989. Des îles dans l'histoire, Paris, EHESS.

—, 2007. La découverte du vrai sauvage et autres essais, Paris, Gallimard.

TAILlEmite Étienne, 1977 (2006). Bougainville et ses compagnons autour du monde. Journaux de navigations établis et commentés par Etienne Taillemite, Paris, Imprimerie nationale.

TCherkézoff Serge, 2005. Tahiti - 1768. Jeunes filles en pleurs. La face cachée des premiers contacts et la naissance du mythe occidental, Pirae (Tahiti), Au vent des îles.

Viveiros De Castro Eduardo, 2009. Métaphysiques cannibales, Paris, PUF.

WaGNer Roy, 1975 (1981). The Invention of Culture, Chicago \& Londres, University of Chicago Press.

3. On pourrait ainsi interroger la manière dont Serge Tcherkézoff analyse le terme " pleurs » en l'isolant de son contexte discursif, et lui donne une importance exagérée, alors qu'on ne trouve qu'une unique occurrence de ce terme dans l'ensemble des journaux. Relisons le passage entier : "Leur mariages se font je crois en public, je tire cette conjoncture de ce qui est arivé peut-être aux deux tiers des françois, des pères et des mères amenoient leurs filles, les présentoient à celui qu’il leur plaisoit, les engageoit à consommer l'œuvre de mariage avec elle. La fille frappoit sur la poitrine de celui à qui elle étoit offerte, lui disant plusieurs mots qui exprimoient suivant le sens que nous leur avons attribué l'abandon qu'elle lui faisoit de sa personne, se couchoit par terre et se dépouilloit de ses vêtements. Plusieurs faisoient des façons quand il s'agissoient d'en venir au fait, se laissoient vaincre cependant. Durant l'opération les assistants insulaires toujour en grand nombre, faisoient un cercle à l'entour, une branche de feuillage à la main, quelque fois ils jettoient sur l'acteur une de leurs toiles, comme à Cythère on couvroit les amants heureux de branches de feuillage. S'il se trouvoient parmis eux quelqu'un qui eut une flutte, il en jouoit, d'autres l'accompagnoient en chantant des couplets dédiés au plaisir. L'opération finie, la fillette pleuroit, se consoloit aisément et faisoit mil caresses à son nouvel époux ainsi qu’à tous ceux qui avoient été spectateurs. " (Journal de Fesche, BCAM, t.II, p.84). Voilà l'apogée de la souffrance dans les récits des relations sexuelles entre marins et Tahitiennes en avril 1768.

4. Par exemple, Serge Tcherkézoff affirme que «l'hypothèse de certains Français sur le désir des jeunes filles ellesmêmes, toujours à la recherche d'amants, est totalement intenable pour le type de rencontres décrites, compte tenu du jeune âge de ces filles" (Tcherkézoff, 2005 : 186) Selon les différentes estimations des marins, ces Tahitiennes avaient entre douze et dix-huit ans. Est-ce impensable de désirer avoir des relations sexuelles quand on a entre douze et dix-huit ans? Que sait-on de la manière dont les Tahitiens considéraient cela? 


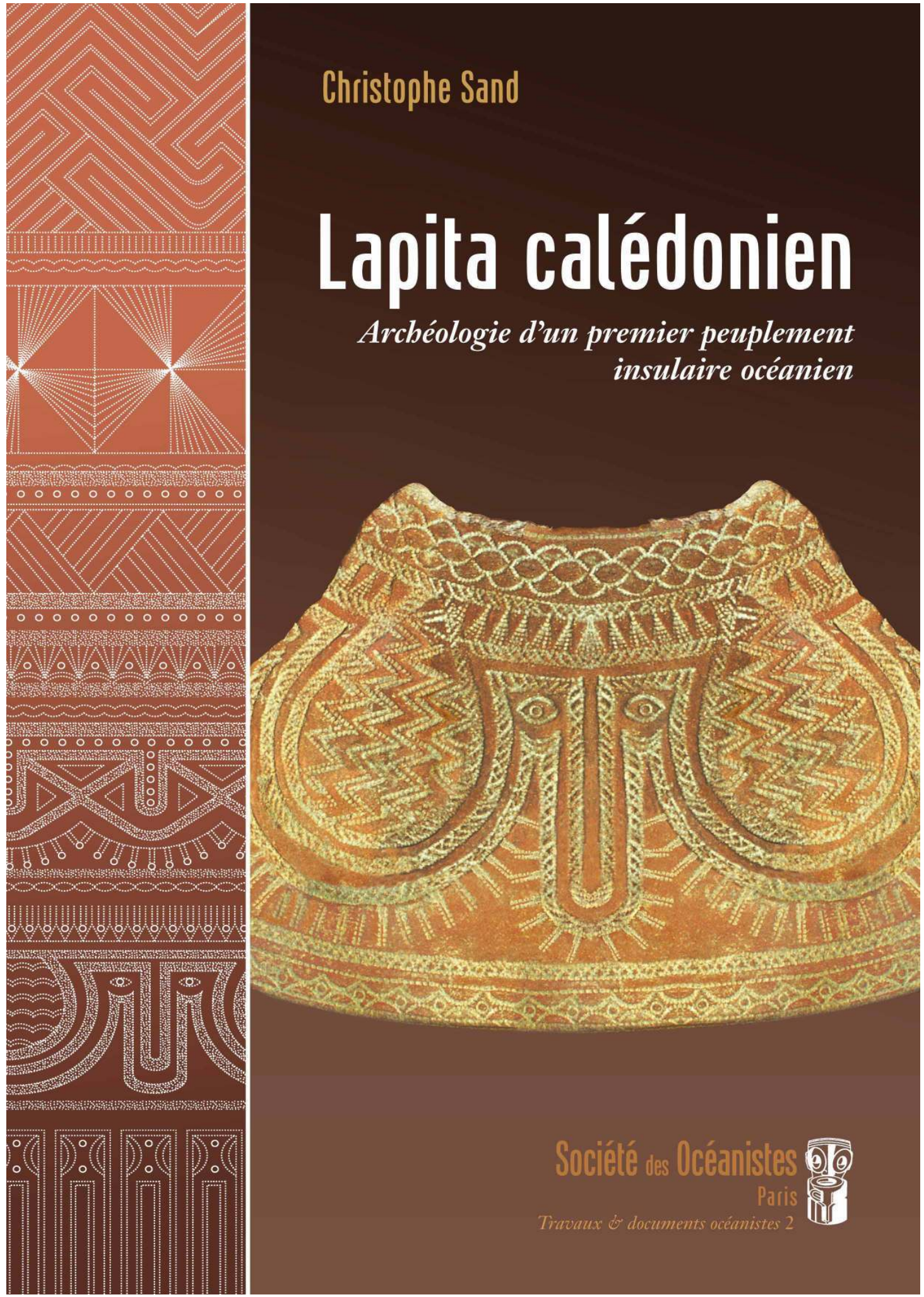

2010, 288 p., bibliographie, index, nombreuses illustrations couleur - $38 €$.

En vente sur http://oceanistes.org/oceanie/spip.php?rubrique29 avec paiement en ligne et en version électronique sur http://books.openedition.org/sdo/1128 - 29,99€. 